Punch Magazine, la construction européenne et une histoire illustrée des relations franco-britanniques de 1950 à 1992

Punch Magazine, European Integration and an Illustrated History of Franco-

British Relations from 1950 to 1992

Niaz Cary-Pernon

\title{
CpenEdition
}

Journals

Édition électronique

URL : https://journals.openedition.org/rfcb/8737

ISSN : 2429-4373

Éditeur

CRECIB - Centre de recherche et d'études en civilisation britannique

Référence électronique

Niaz Cary-Pernon, «Punch Magazine, la construction européenne et une histoire illustrée des relations franco-britanniques de 1950 à 1992 », Revue Française de Civilisation Britannique [En ligne], XXVII-1 | 2022, mis en ligne le 04 janvier 2022, consulté le 01 février 2022. URL : http:// journals.openedition.org/rfcb/8737

Ce document a été généré automatiquement le 1 février 2022

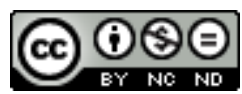

Revue française de civilisation britannique est mis à disposition selon les termes de la licence Creative Commons Attribution - Pas d'Utilisation Commerciale - Pas de Modification 4.0 International. 


\section{Punch Magazine, la construction européenne et une histoire illustrée des relations franco-britanniques de 1950 à 1992}

Punch Magazine, European Integration and an Illustrated History of Franco-

British Relations from 1950 to 1992

Niaz Cary-Pernon

\section{Introduction}

1 Les travaux universitaires sur les relations entre la France et le Royaume-Uni brossent régulièrement le tableau de la coopération, des rivalités et des malentendus qui ont émaillé l'histoire internationale de ces deux pays au vingtième siècle ${ }^{1}$. Pour ce faire, la construction européenne offre un angle d'attaque privilégié. Ses diverses étapes permettent d'exposer, par exemple, les principes justifiant la place que ces deux puissances accordent au Vieux continent dans leurs actions internationales depuis près de soixante-dix ans ${ }^{2}$. Les valeurs diplomatiques et géopolitiques véhiculées dans ce domaine constituent alors des sujets fréquemment observés. Les recherches portant exclusivement sur des sources iconographiques sont peu nombreuses ${ }^{3}$ et le choix archivistique de cet article se fonde sur deux interrogations.

Dans son article paru en 1973 et intitulé "The Cartoon as a Historical Source", l'historien Thomas Milton Kemnitz souligne que Punch Magazine a, depuis de longues années, participé aux débats politiques au Royaume-Uni ${ }^{4}$. Fondé en 1841 , le magazine est sous-titré The London Charivari à ses débuts car il s'inspire directement du Charivari français. Dès sa création, son objectif consiste à dénoncer l'hypocrisie de l'élite de la société victorienne. Cependant, le magazine satirique se fait rapidement l'écho de la voix de l'ordre établi et son lectorat se compose essentiellement de la classe moyenne, 
des couches aisées et des dirigeants britanniques ${ }^{5}$. Au cours du vingtième siècle, Punch occupe une place de choix dans le paysage médiatique au Royaume-Uni. Son tirage atteint près de 175.000 exemplaires dans les années 1940, décennie représentant l'apogée de son succès dans ce domaine $e^{6}$. Dans la deuxième moitié du vingtième siècle, l'expansion d'autres moyens médiatiques, notamment celle de la télévision, a pour effet de réduire l'influence de ce magazine dont les ventes commencent à décliner. Toutefois, la longévité, la renommée mondiale et la diffusion encore significative de Punch continuent à lui conférer un statut respectable et culturellement symbolique à l'échelle tant nationale qu'internationale. Ce n'est qu'à partir de 1978 qu'une baisse considérable de sa distribution survient. Le déclin se poursuit et conduit à l'arrêt de sa parution en 1992. Quatre ans plus tard, l'homme d'affaires Mohamed Al Fayed achète le titre pour relancer l'hebdomadaire, mais en vain; la production cesse définitivement en $2002^{7}$. La baisse notoire des ventes de Punch n'intervient par conséquent que vers la fin de la période d'analyse de cet article.

3 Au regard de tous ces constats, la première interrogation est la suivante : quelle part Punch Magazine prend-il dans le traitement du thème de la construction européenne après la Seconde Guerre mondiale? De plus, la présence de l'acteur français dans les dessins politiques du magazine abordant les actions britanniques face à ce processus historique semble foisonnante. Quelles sont alors les modalités selon lesquelles la France est dépeinte ? Pour étudier ces aspects, l'article appréhende le regard que Punch porte sur les relations franco-britanniques, en se focalisant sur le rôle illustratif, explicatif ou critique des images. Il s'agit de comprendre comment l'élaboration des dessins politiques sur la construction européenne peut façonner au Royaume-Uni une mémoire visuelle et symbolique de ces rapports de 1950 à 1992. Le début de cette borne chronologique se réfère à la parution du premier dessin symbolisant, selon Punch, le point de départ du projet européen; 1992 correspond à la date de la première suspension de la production de cet hebdomadaire en raison de l'essoufflement de ses ventes. Cette étude présente ainsi une perspective d'analyse diachronique, fondée sur une unité thématique, géographique et archivistique. Dans ce cadre, les sources iconographiques sont composées de quarante-neuf dessins politiques ${ }^{8}$, conçus par dix artistes.

Le but de cet article n'est pas de retracer la perception individuelle des dessinateurs pour souligner, par exemple, leurs convictions personnelles. Ce qui importe, c'est l'expression d'une mémoire iconographique longitudinale et globale offerte par Punch dans un domaine de la politique étrangère britannique pour y déceler des évolutions ou des continuités du point de vue iconique et/ou linguistique. Fondant nos réflexions sur les travaux qui appréhendent la pertinence des dessins satiriques pour les historiens ${ }^{9}$, nous postulons que les illustrations politiques de Punch témoignent d'une représentation médiatique de certains aspects relatifs aux mentalités d'un espace et d'une période, espace et période à la fois spécifiques - ceux d'un magazine et d'un pays - et généraux - ceux de la scène politique britannique pendant environ quarante ans. Dans cette perspective, les documents iconographiques deviennent des objets d'étude permettant d'examiner comment un événement historique est commenté et interprété. Ils remplissent par conséquent " une fonction critique », « reflétant un état de l'opinion ou de la culture dont ils sont issus $»^{10}$.

5 À partir de l'inventaire des éléments constitutifs des dessins politiques de Punch et dans une démarche qui procède de l'explicite vers l'implicite, il s'agit d'aborder les choix 
graphiques et thématiques des artistes lorsque ces derniers évoquent les relations franco-britanniques à divers moments de la construction européenne. Une attention particulière est dès lors accordée aux personnages et à leurs fonctions ainsi qu'aux structures symboliques qui permettent de mettre en lumière des représentations collectives et des référents communs en s'appuyant sur la fonction descriptive, la dimension polysémique et la portée critique des images. Pour ce faire, trois axes majeurs guident l'organisation de cet article. Le premier explore les acteurs incarnant les relations franco-britanniques. Le deuxième analyse les références culturelles auxquelles de nombreux dessins font allusion, contribuant dès lors à orienter leur lecture, puisqu'il faut y déceler un sens implicite partagé entre les dessinateurs et les observateurs afin de dépasser les étapes de la représentation et de l'interprétation rudimentaires des événements. Enfin, la troisième partie aborde la manière dont les rapports franco-britanniques sont esquissés dans Punch tout au long de la construction européenne et ce que la présentation qu'en fait le magazine révèle sur l'appréciation du Royaume-Uni de ce processus.

\section{Scènes de vie politiques et sociales}

6 À partir d'une analyse littérale des dessins traitant de la construction européenne, la première étape de cet article consiste à identifier les personnages qui établissent les rapports entre le Royaume-Uni et la France. Cette partie privilégie la fonction descriptive et illustrative des images pour étudier comment les événements sont racontés et quelles fonctions ont les figures dépeintes.

\section{Figures historiques}

7 Jusqu'en 1978, d'après les signes iconiques et linguistiques présents dans notre corpus, les relations franco-britanniques sont d'abord une affaire d'individus. Elles relèvent des initiatives prises par des hommes d'État placés au sommet de la hiérarchie gouvernementale. Dans le contexte britannique, le Premier ministre est le personnage principal mais, comme il en sera question ultérieurement, d'autres protagonistes se trouvent associés à ses actions; en revanche, la France est représentée essentiellement en la personne du général de Gaulle (dix dessins) puis de Georges Pompidou (quatre dessins) et, dans une très moindre mesure, de François Mitterrand (un dessin). La seule occurrence évoquant une personnalité politique française hormis le Président de la République se trouve dans une illustration de Leslie Gilbert Illingworth ${ }^{11}$. Elle renvoie au "plan Schuman", mesure portant le nom du ministre des Affaires étrangères considéré comme le père fondateur de la construction européenne, avec la création de la Communauté du charbon et de l'acier en avril 1951, soit un an après la parution de ce dessin. La référence explicite à Robert Schuman est à la fois linguistique et iconique. Cette image est la première de notre corpus, ce qui signifie que cet événement matérialise, pour Punch, le point de départ du projet européen.

8 Le titre du dessin, le "Pont de la concorde ", est en français. Celui-ci est associé à un processus économique et industriel concernant le charbon et l'acier, terminologies reproduites en toutes lettres sur la passerelle qui s'apprête à être érigée entre la France et l'Allemagne de l'Ouest alors que le Royaume-Uni demeure absent de cette démarche. Cette dernière consiste à parer la "méfiance » et le "soupçon ${ }^{12}$ ", mots écrits sur un 
plan perpendiculaire orientant la lecture du bas en haut et de la gauche vers la droite ; ils sont mis en avant au deuxième plan, notamment par la taille des lettres qui les composent. Ce projet de rapprochement semble donc ne concerner, par nécessité, que la France et l'Allemagne de l'Ouest. L'absence de l'acteur britannique dans ce document tend à refléter la réalité historique. En effet, les autorités britanniques n'ont pas été informées de la préparation du plan Schuman alors que le secrétaire d'État américain, Dean Acheson, et le chancelier allemand, Konrad Adenauer, ont été avertis. Ils ont tous deux accordé leur soutien au projet. En conséquence, la réaction du secrétaire d'État aux Affaires étrangères britannique de l'époque, Ernest Bevin, s'est avérée extrêmement hostile ${ }^{13}$. Dans un contexte de guerre froide, ce dernier était un farouche défenseur d'une politique internationale visant à situer le Royaume-Uni au cœur d'un système de relations privilégiées avec les États-Unis et l'Empire formel et informel/ Commonwealth tout en veillant à consolider la position de son pays sur le continent européen pour contrer la puissance soviétique ${ }^{14}$.

9 Si le processus européen tel qu'il est décrit dans Punch implique régulièrement non seulement la France mais également l'Allemagne, les rapports anglo-allemands se révèlent toujours amicaux. Les chanceliers de la République fédérale d'Allemagne appuient les candidatures britanniques auprès des décideurs français. Ils apparaissent sous les traits d'un conseiller-allié des négociateurs du Royaume-Uni, s'évertuant, par exemple, à encourager le Premier ministre Harold Macmillan à s'engager dans l'aventure européenne en 1961, conseil se traduisant, littéralement et métaphoriquement, par l'invitation à ne pas hésiter à se jeter à l'eau ${ }^{15}$. La bienveillance des protagonistes allemands à l'égard de la Grande-Bretagne est manifeste alors que les relations franco-britanniques se caractérisent par la méfiance et le soupçon, comme le montre un dessin de Norman Mansbridge en $1967^{16}$. Les paroles du général de Gaulle y indiquent que, pour justifier son veto à la candidature britannique à la Communauté économique européenne (CEE), il reproche à Harold Wilson d'agir sous l'influence américaine. Il s'agit là d'une référence directe à une conférence de presse donnée par le président français en 1963. Celui-ci y déclare que le Royaume-Uni agit comme «le cheval de Troie des États-Unis en Europe ${ }^{17}$, ce qui justifie son opposition aux candidatures britanniques à la CEE en 1963 et en 1967. Des transformations de pratiques politiques, stratégiques, commerciales et économiques sont exigées par le gouvernement français. Les missiles américains Polaris dont le Royaume-Uni se dote en 1962 renforcent la méfiance du général de Gaulle ${ }^{18}$.

De plus, tout au long de l'intégration européenne, la représentation des acteurs britanniques est plus nuancée que celle des décideurs français. Dans les dessins figure, par exemple, Geoffrey Rippon, à la tête de la délégation du Royaume-Uni lors de sa troisième candidature dont le processus débute dès décembre $1969^{19}$. À cette occasion, des personnages sans visage incarnent la catégorie des technocrates étudiant diverses questions extrêmement précises, qui ne semblent pas attirer l'attention du Premier ministre Edward Heath ${ }^{20}$. La présence de ces figures anonymes tend à souligner que ce ne sont pas les hommes politiques, issus d'un système démocratique représentatif, mais des experts administratifs qui portent la responsabilité de l'examen de décisions affectant le peuple britannique. La notion de la souveraineté parlementaire, fondement constitutionnel du régime politique en Grande-Bretagne, se trouve au cœur d'un système de représentations qui, selon certains analystes, explique la réticence des 
décideurs britanniques envers la Communauté européenne considérée comme un colosse supranational menaçant l'identité politique de leur pays ${ }^{21}$.

11 Le regard plus complexe porté sur les acteurs londoniens peut se justifier. Les dessinateurs de Punch analysent la politique européenne du Royaume-Uni; les protagonistes des autres pays désignent surtout, par synecdoque, les nations dans lesquelles ils exercent leur fonction. Toutefois, nombre de dessins mettent en lumière la personnalité des présidents français en se focalisant, par exemple, sur l'attitude positive de Pompidou, affichant un large sourire ${ }^{22}$. Grâce à lui, la Grande-Bretagne peut enfin espérer participer au projet européen. En revanche, insister sur la personnalité du général de Gaulle, toujours présenté comme intransigeant et obtus, constitue l'un des principaux leitmotive ${ }^{23}$. La mise en images de son hostilité dans Punch signale un obstacle récurrent que les Premiers ministres britanniques engagés dans le processus européen de cette époque, Macmillan et Wilson, ne parviennent pas à franchir malgré les efforts fournis, parfois au péril de leur carrière sur le plan de la politique intérieure $^{24}$. En effet, les négociations sur la troisième candidature britannique pour entrer dans la CEE sont entamées sous le gouvernement Wilson, avec l'aval très réservé du Parti travailliste, et l'adhésion est finalement conclue par l'administration du Conservateur Heath. Dès lors qu'il n'exerce plus le pouvoir, le Parti travailliste va affirmer une opposition farouche aux termes négociés ${ }^{25}$.

12 Ainsi, jusqu'à la participation du Royaume-Uni à la CEE, l'appréciation de l'exécutif français sur les projets d'outre-Manche semble primordiale. Selon Punch, la rigidité et les opinions personnelles du général de Gaulle, soit l'attitude d'un seul décideur, expliquent largement l'échec des candidatures britanniques jusqu'au moment où la fonction présidentielle se trouve incarnée par un nouveau responsable à l'Élysée. Cette interprétation est largement corroborée par les travaux universitaires cités. L'importance accordée à de Gaulle met en avant un projet européen contrôlé par un régime politique français qui est caractérisé comme hyper-présidentiel. De surcroît, bien plus qu'un décideur, le président français est une figure d'envergure internationale, extrêmement connue du lectorat britannique.

\section{Figures sociales}

13 L'adhésion du Royaume-Uni à la CEE sous la présidence de Pompidou introduit un changement radical quant à la nature des acteurs dépeints. Désormais, le nombre des dessins mettant en scène le peuple britannique excède largement celui des illustrations centrées sur les hommes politiques. Sur un total de quinze images pour la période 1973-1992, on dénombre onze occurrences avec des acteurs sociaux. Ces derniers occupent exclusivement le paysage dans les années 1980-1992. Les actions des décideurs londoniens s'apprécient dorénavant indirectement, à travers des commentaires s'y rapportant par l'intermédiaire de la vox populi. Celle-ci revêt différentes formes et peut être associée à des marqueurs de la classe sociale. S'expriment, par exemple, des couples ou des supporters du football britanniques en France. On observe également les réactions de certains citoyens ordinaires se trouvant chez eux ou dans un pub ${ }^{26}$.

14 D'une manière générale, pendant les périodes d'avant- ou d'après-1973, l'appréciation de l'opinion publique britannique sur la construction européenne s'avère largement critique. Pour des touristes en France ou pour un homme appartenant à la couche 
supérieure de la société, les mesures européennes se définissent d'abord par leur absurdité ${ }^{7}$. Elles s'appliquent, car des technocrates l'ont décidé et non parce qu'elles sont justes et nécessaires ou bien parce qu'elles améliorent le quotidien des Britanniques. Dans les dessins, la France incarne ce système technocratique décrié. Par conséquent, dans un restaurant, un client choisit de manger ce que la CEE peut offrir en grande quantité et non ce qu'il souhaite véritablement déguster. La surproduction agricole européenne dicte son comportement alimentaire. Son choix peut également mettre en exergue son souhait de profiter du système européen alors qu'il se trouve dans un restaurant de qualité lui offrant l'occasion de consommer des produits plus rares et raffinés ${ }^{28}$. Ce renversement des valeurs est corroboré dans les paroles de deux représentants du monde agricole qui sont rémunérés non en exerçant leur métier mais en «pomp[ant] des subventions européennes ${ }^{29}$ ".

Dès lors, toutes les catégories socio-professionnelles du peuple britannique dans les dessins adoptent un comportement similaire. Les signes linguistiques transcrivant leurs paroles révèlent, au mieux, leur incompréhension vis-à-vis des jeux politiques européens et, au pire, leur volonté de signaler que les mesures européennes leur semblent injustes ou dénuées de sens, essentiellement à cause de l'influence française ${ }^{30}$. Dans ces circonstances, les personnages évoquant le peuple français se muent en contre-exemples. Les Britanniques s'y réfèrent pour commenter, voire critiquer, les aberrations induites par les divers organismes européens ${ }^{31}$. Toutes ces objections rappellent l'attitude extrêmement virulente de la presse tabloïde, jouissant d'un vaste lectorat britannique, envers la construction européenne ${ }^{32}$. Elles reflètent également les résultats des sondages menés, par exemple, par la Commission européenne dans les États membres grâce à l'outil d'analyse l'Eurobaromètre. Au Royaume-Uni, l'opinion publique manifeste régulièrement son hostilité envers l'intégration européenne ${ }^{33}$.

Dans Punch, le peuple britannique représenté dans ses différentes facettes incrimine les décideurs français, alléguant que le processus d'intégration auquel le Royaume-Uni s'est désormais associé privilégie les pays fondateurs du projet. Les organismes ne les représentent pas, car ils servent des intérêts avant tout français mais également allemands et italiens ${ }^{34}$. Les règles du jeu sont par conséquent perçues comme déloyales et inégalitaires. Le comportement français paraît particulièrement inadmissible. L'opinion publique britannique, telle qu'illustrée dans Punch, estime en effet que c'est grâce à son propre héroïsme et aux initiatives prises par son propre gouvernement pendant la Seconde Guerre mondiale que les pays engagés dans la construction européenne, et plus précisément la France, sont en mesure d'élaborer des programmes communs ${ }^{35}$. Or, pour les lecteurs du magazine, la fabrique événementielle du processus européen retrace les humiliations essuyées par Macmillan et Wilson à cause du général de Gaulle ${ }^{36}$. Ici s'exprime la culture populaire qui s'appuie sur un imaginaire historique valorisant le rôle que la Grande-Bretagne a joué face au totalitarisme. Selon cette perception héroïque, contrairement à nombre de pays de l'Europe continentale, le Royaume-Uni n'a pas subi l'invasion allemande ni collaboré avec le régime nazi. On note également une référence directe au prestige conféré à la ville de Londres en tant que siège de la France libre dirigée par le général de Gaulle ${ }^{37}$. L'attitude de celui-ci semble alors d'autant plus ingrate, selon Punch.

17 Ainsi, dans Punch, la participation du Royaume-Uni à une initiative essentiellement franco-allemande relève de la volonté de quelques Premiers ministres, assistés de quelques hommes d'État ou technocrates. Puis, lorsque le pays adhère à la CEE, les 
dessinateurs dressent essentiellement le bilan et l'impact de cette aventure à travers la vox populi. Toutefois, si les décideurs et les administrés ne portent pas nécessairement le même regard sur les diverses étapes de la construction européenne, l'attitude des acteurs français est une référence constante, concourant à justifier les échecs et les succès des hommes politiques ou bien les difficultés rencontrées par le peuple au Royaume-Uni. En outre, dès l'adhésion britannique à la CEE, la substitution des personnalités politiques par des figures qui incarnent l'opinion publique attire l'attention sur la fonction explicative des images, permettant de saisir la complexité d'un processus historique aux multiples facettes.

\section{Réalités polymorphes}

Les lecteurs de Punch doivent régulièrement mobiliser des connaissances culturelles pour mieux appréhender la signification des dessins politiques sur les relations francobritanniques au cours de la construction européenne. Les dessinateurs guident ainsi le décodage des événements, invitant les observateurs à s'interroger sur la polysémie des images afin de dépasser la simple compréhension des réalités événementielles exposées.

\section{Symboles stéréotypés}

19 L'un des procédés majeurs utilisés dans Punch relève des symboles stéréotypés, terme entendu comme une image collective simplifiée et figée qui vise à réduire les particularités. En effet, les acteurs politiques britanniques sont souvent dotés de plusieurs fonctions emblématiques. Par exemple, Macmillan, essuyant l'échec des négociations sur l'entrée du Royaume-Uni dans la CEE en 1963 à cause du refus français, apparaît sous les traits d'une femme en désarroi ${ }^{38}$. À ces caractéristiques liées au genre, d'autres références culturelles peuvent se juxtaposer, convoquant la notion d'implicite et renvoyant au répertoire classique, littéraire ou historique, du grand public. Concernant les références littéraires, le Premier ministre Macmillan est caricaturé en Cendrillon dans un dessin d'Illingworth en 1962. Cela permet d'afficher la cruauté de ses demi-sœurs, incarnées par de Gaulle et Adenauer ${ }^{39}$. Macmillan se révèle ainsi soumis à des personnages dont le portrait parodique charge l'impitoyable de Gaulle, dépeint comme la principale cause de sa misère. Cette idée sous-tend un autre dessin $\mathrm{d}^{\prime}$ Illingworth ${ }^{40}$. De Gaulle est cette fois déguisé, par zoomorphisme, en méchant loup demandant au Petit chaperon rouge - Wilson - d'avancer. Le danger d'être dévoré par la France serait-il imminent pour le Royaume-Uni? Telle est la question soumise aux observateurs.

De même, lorsque la seule référence à Margaret Thatcher dans Punch s'effectue, de façon parodique, par l'intermédiaire d'un épisode d'Alice au pays des merveilles ${ }^{41}$, l'objectif est de souligner - par ironie et en référence à un dessin de John Tenniel, principal illustrateur de l'œuvre de Lewis Carroll à laquelle il est fait allusion - que la Tortue allemande et le Griffon menaçant français, loin de se présenter comme des amis, semblent effrayer Alice-Thatcher. Celle-ci ne parvient pas à entrer dans la danse européenne avec les géants Helmut Kohl et François Mitterrand. Cette esquisse de Thatcher, apeurée, contredit, par exemple, son âpre combat pour réduire la contribution britannique au budget européen en $1984^{42}$. Cette représentation peut, tout 
au plus, révéler son euroscepticisme. Selon ce dessin, la France et l'Allemagne symbolisent désormais la menace suscitant la crainte de la Dame de fer, ce qui amoindrit quelque peu l'omniprésence, dans notre corpus, de la responsabilité néfaste $\mathrm{du}$ président français à l'égard du Royaume-Uni. Ainsi, cette image indique que, face à l'alliance des géants français et allemand des années 1980, Thatcher est affaiblie et ses pouvoirs européens rétrécis comme Alice. Au-delà de ses convictions eurosceptiques, elle ne parvient pas à faire le poids face à Mitterrand et Kohl, au sens propre comme au figuré.

De plus, dans des contextes brossant souvent un environnement tendu pour les acteurs politiques, on décèle de nombreuses allusions à des épisodes historiques évoquant les heures glorieuses de la diplomatie du Royaume-Uni alors que la France était tombée sous le joug du nazisme. Toutefois, ces références sollicitent une lecture ironique, car les allusions viennent régulièrement désavouer les efforts et le courage que les Premiers ministres britanniques impliqués affichent. Par exemple, dans un dessin de Mansbridge en $1967^{43}$, reprenant le scénario de David Low sur la défaite militaire de la France en $1940^{44}$, les paroles de Wilson signalent qu'il désire se présenter comme un héros de la Seconde Guerre mondiale lors de la deuxième candidature britannique en 1967. Mais ses paroles dévoilent une résonance tragique. En effet, l'étude de cette candidature sera fortement influencée par le regard du général de Gaulle et les lecteurs de Punch connaissent parfaitement ses convictions, ne faisant aucun mystère de son opposition. En filigrane, se lit alors la forte probabilité que cette candidature soit contrée précisément par l'homme politique accueilli et soutenu par Londres pendant la Seconde Guerre mondiale.

Ainsi, dans les allusions, les symboles féminins sont associés à des moments de détresse et de faiblesse dont les hommes politiques britanniques font l'expérience sur le chemin de la construction européenne. En revanche, le courage face à l'adversité nationale et internationale est surtout illustré par des images de guerriers, fonction hautement masculine, même si les références aux épisodes de la Seconde Guerre mondiale dénotent une résonance tragiquement ironique. Elles sapent, à leur tour, la mise en images du courage des acteurs politiques, revêtant dès lors des accents ridicules.

Au niveau d'une lecture plus nuancée des thèmes d'actualité européenne dans Punch, la deuxième catégorie de symboles stéréotypés mobilise des emblèmes nationaux. Parmi ces derniers, c'est essentiellement John Bull qui incarne le Royaume-Uni lorsqu'un représentant précis de la classe politique ou de l'opinion publique ne constitue pas le point central ${ }^{45}$. L'appréciation de ce paramètre allégorique n'est toutefois pas tant quantitative que qualitative. Son émergence est étroitement associée à l'interaction, voire à la compétition, entre les souverainetés nationales de la France et du RoyaumeUni. Par exemple, dans un dessin de Mansbridge ${ }^{46}$, Marianne interdit à John Bull l'entrée dans un bus en direction de la zone de libre-échange ${ }^{47}$ et du Marché commun.

Dans ce document, à gauche, aux deuxième et troisième plans, Marianne est accompagnée de deux silhouettes du général de Gaulle. Il est ainsi suggéré que le système politique français se caractérise par un régime présidentiel dans lequel le chef de l'exécutif s'avère être le seul décideur. Cela rappelle un dessin de Low sur la conception de l'Europe du général de Gaulle ${ }^{48}$. En juxtaposant les deux images, on peut affirmer que, selon Punch, la France et la Communauté européenne sont dirigées par de Gaulle. Par ailleurs, le dessin de Mansbridge établit clairement que les deux projets dans lesquels John Bull semble impliqué fragilisent ses relations avec le 
Commonwealth ${ }^{49}$. En outre, dans la représentation du processus européen, ce sont essentiellement des symboles du folklore anglais qui se trouvent réactivés par les dessinateurs ${ }^{50}$. Dans ce contexte d'appréciation symbolique portée sur les événements dépeints, une question s'impose légitimement: les portraits à charge se limitent-ils seulement aux décideurs français? Les Britanniques, tels qu'ils sont décrits dans Punch, ne révèlent-ils pas également leur part d'ambiguïté ou de faiblesse?

\section{Responsabilités partagées}

En 1988, un dessin de David Langdon évoque, de façon ironique, que des Britanniques se ruent vers la France pour bénéficier des produits détaxés ${ }^{51}$. Pourtant, dans deux autres illustrations de cet artiste, la France incarne, selon ces personnages sociaux, tous les travers administratifs et économiques de la $\mathrm{CEE}^{52}$. L'Hexagone revêt dès lors une fonction qui tient lieu de repoussoir tandis qu'il se présente parallèlement comme une destination de prédilection pour des activités de loisir et de tourisme. Le peuple britannique n'est donc pas exempt de tout reproche et expose sans vergogne des signes d'incohérence, ne tenant compte que de ses propres avantages tantôt financiers tantôt touristiques. En outre, avant ou après 1973, l'opinion publique exprime son manque d'intérêt pour les affaires européennes, préférant, par exemple dans un dessin de Trog en 1971, regarder un match de football à la télévision alors que le Premier ministre Heath s'engage, tel le chevalier St Georges, dans la bataille de la troisième candidature du Royaume-Uni à la $\mathrm{CEE}^{53}$. Ainsi, cette bataille n'est pas soutenue par le peuple britannique, qui plus est, comme nous l'avons souligné précédemment, affiche ouvertement son aversion à la question européenne. Selon Punch, un élément d'explication de ce phénomène consiste à évoquer la responsabilité des décideurs. Ces derniers montrent leur incompétence en ne parvenant pas à sortir le Royaume-Uni de ses difficultés économiques, au-delà de la solution que le processus européen est censé constituer ${ }^{54}$.

Dans Punch, les décideurs londoniens semblent en effet reconnaître que leur pays devrait participer à une aventure européenne, menée par la France, tout en montrant de nombreuses indécisions et discordances. Ces thématiques sont particulièrement mises à l'honneur lorsqu'il s'agit de commenter la position du Parti travailliste. D'un point de vue global, si l'on considère la mémoire visuelle véhiculée par Punch sur la construction européenne, les références historiques, renvoyant notamment à l'épisode de la Seconde Guerre mondiale, tendent à décrédibiliser l'héroïsme de Wilson lors de la deuxième candidature britannique ${ }^{55}$ : parallèlement à tous les risques qu'il est prêt à prendre, son parti paraît souvent divisé et incohérent sur la question de l'engagement du Royaume-Uni dans la $\mathrm{CEE}^{56}$. Ainsi, le veto français, incarné essentiellement par de Gaulle, n'est pas la seule cause des déboires britanniques. La mésaventure en 1963 relèverait également de la responsabilité des chefs politiques, puisqu'aucune autre alternative ne semble prendre forme en cas d'échec ${ }^{57}$.

L'analyse des images dans Punch invite par conséquent les observateurs à aller au-delà de la simple compréhension événementielle des diverses étapes historiques. Même si les décideurs français sont régulièrement évoqués, directement ou indirectement, pour justifier les réactions des représentants politiques et sociaux au Royaume-Uni, les documents iconographiques ne manquent pas de souligner, comme les analyses universitaires ${ }^{58}$, que l'aventure européenne a constitué une solution par défaut pour les 
instances londoniennes, suscitant également des divisions et des incohérences au sein du Parti travailliste ${ }^{59}$.

\section{Quelle grammaire visuelle du positionnement européen du Royaume-Uni ?}

Dans cette partie, il s'agit d'appréhender ce que la représentation des relations francobritanniques dans Punch permet d'affirmer à propos du positionnement européen du Royaume-Uni. Comment les images encouragent-elles à porter un jugement sur le comportement de tous les acteurs alors qu'un nouvel espace européen se façonne, contraignant les décideurs britanniques à y exprimer leurs projets et points de vue ?

\section{Identités asymétriques}

Le portrait de la vision critique des figures sociales britanniques vis-à-vis de la construction européenne conduit les observateurs à examiner la nature et la pertinence de ce processus. En effet, dans Punch, l'esquisse de l'identité internationale du Royaume-Uni est plurielle et complexe. Le terme identité signifie ici la façon dont le pays est défini et dépeint sur la scène internationale. Par exemple, alors qu'à l'échelle mondiale, le profil colonial de la Grande-Bretagne est comparable à celui de la France, pour celle-ci, la question européenne demeure, dans les dessins, une problématique purement française. En revanche, les relations internationales du Royaume-Uni sont déclinées par Punch sous des aspects qui font écho à l'interdépendance entre les trois cercles churchilliens, ce qui, précisément, constitue pour le gouvernement français une entrave majeure lors des candidatures britanniques dans les années $1960^{60}$.

Dans cette configuration, en examinant le poids accordé à chaque sphère churchillienne, on remarque que peu de dessins abordent la relation spéciale sauf quand il s'agit d'évoquer l'attitude du général de Gaulle ${ }^{61}$. Ce sont surtout les interactions entre le Royaume-Uni et le Commonwealth qui se trouvent esquissées. À l'instar des arguments avancés dans certains travaux universitaires, le Commonwealth semble s'ériger comme une référence géographique, symbolique et institutionnelle, offrant un cadre d'analyse des réticences britanniques à la construction européenne ${ }^{62}$. En effet, l'incompréhension et/ou la colère des partenaires du Commonwealth à l'égard du comportement des chefs de file londoniens sont des thèmes récurrents, indiquant que l'aventure européenne se réalise aux dépens des traditions, bien ancrées selon Punch, de la politique étrangère britannique ${ }^{63}$. L'engagement européen, exigeant une identité internationale exclusive et restreinte, devient alors synonyme de chaos comme le symbolise l'attitude de Macmillan dans un dessin d'Illingworth en $1962^{64}$. Dans cette illustration, le Premier ministre britannique est assis sur une falaise, aux deux-tiers de sa hauteur, et fait face à des représentants du Commonwealth, se tenant debout sur la terre ferme et commentant la scène qui se déroule sous leurs yeux; les décideurs politiques Adenauer et de Gaulle se situent au sommet de la falaise, débattant sans prêter attention à l'environnement général. Le président français semble indifférent face aux difficultés rencontrées par Macmillan.

31 En outre, la corde permettant à ce dernier de terminer son ascension pour se positionner, à son tour, au point culminant de la falaise est tenue par le général de Gaulle. Or, la position de cette corde empêche le représentant britannique d'y avoir 
accès pour rejoindre les hommes politiques français et allemand. Ainsi, le chemin européen emprunté par Macmillan, au lieu d'enrichir la situation du Royaume-Uni à l'échelle nationale et mondiale, sème le désordre, la peur et l'épuisement ainsi que le suggèrent son visage et son attitude générale. Macmillan, ayant entrepris la démarche d'être aux côtés de la France et de l'Allemagne de l'Ouest en haut de la falaise, fait alors face aux représentants du Commonwealth en leur lançant un appel au secours en français après l'avoir fait en anglais ${ }^{65}$.

32 L'observateur peut se demander si cet appel du représentant britannique esseulé s'adresse à la France afin d'être sauvé des griffes des émissaires du Commonwealth ne paraissant pas particulièrement bienveillants à son égard ou bien si cette démarche linguistique ne dénote pas une erreur stratégique. Macmillan, dans un moment de panique, confondrait alors les contextes en prononçant des paroles en français à l'intention du Commonwealth. À moins qu'employer la langue de Molière dans une sphère anglophone ne constitue une preuve de sa bonne volonté sur la scène internationale afin de plaire à de Gaulle. Rappelons également le retrait de l'Afrique du Sud du Commonwealth en 1960, action qui fait voler en éclats la force de ce référent traditionnel de la politique étrangère britannique de l'époque. Toutes ces possibilités interprétatives induites par des techniques illustratives utilisant des procédés d'opposition explicites et implicites incitent ainsi à s'intéresser à une lecture métaphorique des dessins politiques de Punch.

\section{Considérations métaphoriques}

33 D'une manière générale, les relations franco-britanniques sont décrites comme inégalitaires. Cette dissymétrie donne clairement l'avantage stratégique, économique et politique à la France. Les décideurs londoniens craignent les présidents français ou doivent fournir des efforts considérables pour pouvoir les satisfaire ${ }^{66}$. Dans ce cadre, les références culturelles que constituent le Petit chaperon rouge, Cendrillon ou bien Alice au pays des merveilles ${ }^{67}$, d'une part, et l'abondance des techniques de contre-plongée ou de plongée dans les dessins selon que le regard des lecteurs est placé du côté français ou britannique ${ }^{68}$, d'autre part, s'avèrent significatives. En effet, les personnalités marquantes lors des candidatures britanniques sont souvent dépeintes dans une position de candidats faibles et/ou victimes devant pourtant affronter les obstacles dressés par la France. La répétition de ce thème de l'infériorité du RoyaumeUni, sans nécessairement susciter la sympathie de l'observateur pour l'acteur britannique, tend néanmoins à engendrer une certaine hostilité envers les représentants français. Cette récurrence thématique dans Punch établit, directement ou indirectement, l'intransigeance et le manque de gratitude des décideurs français envers un peuple allié et protecteur pendant le dernier conflit mondial, ainsi que le souligne la vox populi étudiée précédemment.

Dans ce contexte, on constate que la position de Winston Churchill vis-à-vis du projet européen demeure absente du corpus de cet article. Cette icône politique apparaît dans un seul dessin, publié en $1971^{69}$. On y voit les fantômes de Churchill et du général de Gaulle, qui, à cette date, sont décédés. Cette illustration est la seule qui esquisse l'attitude humble et soumise de l'ancien Président de la République française. Sa composition souligne que celui-ci, se tenant tête basse, semble vaincu par précisément l'homme politique britannique qui lui a permis de sauver la France dans les heures les 
plus sombres de son histoire. Désormais, de Gaulle ne peut plus imposer son veto à la candidature britannique, ce qui représente un avantage stratégique pour le Premier ministre Heath. Mais le fantôme français est surtout placé derrière celui de Churchill affichant un visage grave. Malgré sa taille qui dépasse celle de Churchill, de Gaulle n'ose pas intervenir. Cependant, Churchill décédé, apprécie-t-il les négociations sur l'entrée de son pays dans la CEE ou bien s'évertue-t-il à protéger le Royaume-Uni lors de sa troisième tentative? L'observateur peut légitimement se poser de telles questions devant ce dessin de Trog et, dans l'ensemble du corpus, il semblerait que Churchill vivant ne puisse faire l'objet d'une représentation à l'égal des autres protagonistes.

De même, l'absence d'un autre Premier ministre britannique, également homme politique de premier rang pendant la Seconde Guerre mondiale, est notable. Il s'agit d'Anthony Eden, qui a pourtant entrepris, dans les années 1955-1957, une réorientation de la politique internationale du Royaume-Uni avec un projet aboutissant à l'Association européenne de libre-échange en janvier 1960 et visant à concurrencer le Marché commun. Par ailleurs, alors que les années 1980 sont marquées par les gouvernements successifs de Thatcher, celle-ci n'apparaît que dans un seul dessin et, comme indiqué ci-dessus, de façon allégorique. Or, le rôle central que cette figure politique a joué dans la modification de la position du Parti conservateur sur la construction européenne a été souligné par les historiens ${ }^{70}$. Un examen rapide des dessins de Punch mettant en scène Thatcher a révélé que ce sont les thèmes de sa politique intérieure qui ont attiré l'attention des artistes. Plus généralement, tous les non-dits ou les allusions furtives dans notre corpus conduisent non seulement à procéder à une interprétation métaphorique des relations franco-britanniques lors de la construction européenne mais également à déceler des remarques critiques sur le positionnement européen du Royaume-Uni.

36 À plusieurs reprises, le rapprochement avec l'Europe est symbolisé dans Punch par le truchement du thème de l'union conjugale, notamment avec la France. Toutefois, au sens littéral ou figuré, cette union se révèle être de circonstances et non un mariage d'amour ${ }^{71}$. L'avenir d'une telle alliance se manifeste souvent comme incertain, ne présageant rien d'heureux ainsi que le suggèrent, dans un dessin de Mansbridge, non seulement le visage et l'attitude des jeunes mariés mais également l'air triste de tous les hôtes $^{72}$. Le rapport anglo-européen peut aussi renvoyer à une liaison dangereuse synonyme de trahison dans un scénario classique exposant un adultère. Dans un autre dessin de Mansbridge en $1961^{73}$, John Bull affiche un comportement hasardeux et risqué lorsque le Royaume-Uni décide officiellement de donner la priorité au Marché commun en sachant que cette action fragiliserait ses relations avec le Commonwealth. Dans cette illustration, le zigzag des courbes des transactions présentées sur le mur de l'entreprise, "John Bull trading company », et l'adultère commis par son chef témoignent de la perfidie britannique et des risques financiers que le rapprochement angloeuropéen induit.

En effet, pour John Bull, la jeune maîtresse européenne assise sur ses genoux est plus attrayante que sa partenaire, plus âgée, nommée Commonwealth. Ce chef d'entreprise infidèle serait donc séduit par les avantages économico-financiers qu'offre l'aventure européenne. L'Europe est cependant personnifiée par une jeune femme qui manifeste des traits physiques semblables à ceux de la femme d'âge plus mûr incarnant le Commonwealth. Les deux figures féminines portent le même type de coiffure; leur tailleur et leurs chaussures sont également similaires. Elles se distinguent 
essentiellement par leur âge ${ }^{74}$. Ce dessin soulève des questions et des commentaires peu élogieux de la part de l'observateur : John Bull serait-il uniquement aveuglé par les apparences et les promesses d'une relation avec une partenaire plus jeune? Peu fiable, n'abandonnerait-il pas cette jeunesse pour un autre type d'alliance dans quelques années dès lors que des occasions plus intéressantes se présenteront? John Bull a l'air surpris et gêné que l'existence de sa liaison avec la maîtresse européenne soit révélée à l'incarnation du Commonwealth. Peu digne de confiance, il n'a pas le courage d'assumer ses actes, à moins qu'indécis, il ne veuille se satisfaire de cette relation triangulaire, ce qui est le cas lorsque le Royaume-Uni décide de présenter sa candidature au Marché commun en 1961, soit juste après avoir contribué activement à la création de la zone de libre-échange concurrençant ce Marché. Ainsi, l'aventure européenne du Royaume-Uni s'avère un choix fondé sur des considérations essentiellement économiques et financières visant à renforcer le statut international du pays grâce à de nouvelles relations européennes ${ }^{75}$.

Par ailleurs, l'expression visuelle du processus européen renvoie d'abord à une entité géographique évoquée par des cartes, des drapeaux ou des visages politiques ${ }^{76}$ pour devenir progressivement une idée abstraite et surtout un géant technocratique dénué d'humanité, symbolisés par des objets transnationaux tels qu'une voiture, un bâtiment en fil de fer ou un navire ${ }^{77}$. Cela fait écho à la présence des figures technocratiques étudiant diverses questions européennes à l'échelle nationale dès lors que le RoyaumeUni adhère à la Communauté européenne en $1973^{78}$. D'après les caractéristiques récurrentes dans les représentations des relations franco-britanniques dans Punch, l'observateur est donc en droit de s'interroger sur la pertinence de la démarche britannique dans un environnement général hostile et incohérent. Cela évoque également la méfiance que l'opinion publique britannique ne cesse d'afficher à l'égard des institutions de la CEE dans les sondages ou bien les réticences que la classe dirigeante londonienne n'a cessé d'exprimer à un projet européen défendant une structure supranationale considérée comme une menace pour la souveraineté britannique ${ }^{79}$.

39 En résumé, lorsqu'il s'agit de commenter l'engagement européen d'outre-Manche, les dessins illustrent régulièrement l'animosité des décideurs français, la colère et l'incompréhension des représentants politiques du Commonwealth face à l'abandon ou la négligence dont ils se sentent victimes ou encore la réticence des figures sociales et politiques britanniques à l'engagement européen de Londres. De plus, l'union européenne s'apparente à une configuration conjugale tantôt polygamique tantôt victimaire, avec des fiancées, des épouses et des maitresses qui inspirent ou suscitent la détresse, le désintérêt et des obstacles, d'une part; cette union renvoie à une association politique et économique hyper-présidée par les décideurs français et dont les organismes représentatifs sont déshumanisés, d'autre part. Cette alliance angloeuropéenne peut-elle alors accorder un rôle significatif à Londres sur le Vieux continent et sur la scène internationale? Cette interrogation sous-tend nombre d'images du corpus de cet article.

\section{Conclusion}

Dans Punch, l'étude typologique des personnages incarnant les relations francobritanniques au cours de la construction européenne permet d'identifier trois grandes 
catégories. La première renvoie aux personnalités du réel, c'est-à-dire aux principaux acteurs historiques présents dans les différentes étapes du projet européen; le deuxième ensemble désigne des entités sociales plus ou moins élargies; enfin, la dernière catégorie se définit davantage par les valeurs symboliques complémentaires associées aux personnages, incitant explicitement les lecteurs à une interprétation nuancée des actions. Un paramètre chronologique joue un rôle significatif : à partir de 1973, lorsque le Royaume-Uni parvient enfin à entrer dans la CEE après sa troisième tentative, on assiste progressivement à une inversion des rapports entre les acteurs politiques et sociaux dépeints.

41 À un premier niveau de lecture, les choix des décideurs politiques et la vox populi britannique s'opposent régulièrement dans les représentations événementielles de la construction européenne. Le peuple ne cesse de relever les conséquences négatives de l'adhésion britannique dans sa vie quotidienne et professionnelle. De plus, les actions des décideurs politiques tendent à souligner le danger auquel l'aventure européenne expose le Royaume-Uni. Tous ces commentaires implicites sur le projet européen encouragent, directement ou indirectement, à aborder la mémoire symbolique que les dessins de Punch véhiculent dans le cadre des relations franco-britanniques. En effet, certains procédés influencent grandement l'appréhension des événements exposés.

Selon un schéma antagoniste et dissymétrique, le comportement des présidents français justifie largement l'échec ou le succès des actions britanniques. Toutefois, les dessins construisent également une réalité qui reflète un regard critique et nuancé à plus d'un titre. Les dessinateurs introduisent souvent une dimension complémentaire, s'évertuant à examiner, à l'aide de symboles parfois divergents, les comportements des figures politiques et sociales britanniques. Ils proposent une analyse journalistique, fondée sur des arguments et des contre-arguments, et une explication réaliste évoquant les raisons pragmatiques qui poussent les Britanniques à réagir face aux projets européens. Ce pragmatisme affiche, essentiellement de façon ironique, des incohérences et la mémoire visuelle de Punch se révèle réprobatrice envers tous les acteurs représentés, politiques et sociaux, britanniques et français.

Cette perception est particulièrement manifeste dans les dessins mettant en avant le principal dilemme qui se présente aux décideurs britanniques. Ces derniers doivent choisir entre un ensemble de pays situés sur l'échiquier mondial - avant tout le Commonwealth - et un espace géopolitique en cours de construction sur le Vieux continent. Cette démarche paraît exclusive puisqu'elle conduit les décideurs à négliger leurs relations avec le Commonwealth, relevant pourtant, selon Punch, d'une tradition diplomatique solidement ancrée dans l'histoire du pays, pour endosser le rôle du bon élève européen. Mais à travers la représentation de l'opinion publique, la répétition de l'argumentation selon laquelle le sort britannique se trouve détérioré, notamment à cause du poids démesuré du rôle de la France dans les institutions transnationales, tend à témoigner d'un environnement politique et social hostile au projet européen, interpellant dès lors le bien-fondé d'un processus décrit comme technocratique et déshumanisé. 


\section{Archives}

Punch Cartoon Library, hébergée par TopFoto et consultée en mars 2018. URL : http://

www.topfoto.co.uk.

\section{Références}

ALEXANDRE-COLLIER Agnès, « Le phénomène eurosceptique au sein du parti conservateur britannique », Politique européenne, vol. 2, no. 6, 2002, p. 53-73.

BBC [En ligne], 24 mars 1992, « On this Day », « Punch ends 150 years of satire », consulté le 4 avril 2018. URL : http://news.bbc.co.uk/onthisday/hi/dates/stories/march/24/ newsid_2531000/2531249.stm.

BELL Michael Hett, France and Britain, 1940-1994: The Long Separation, Londres, Routledge, 1997.

CHAOUAD Robert, « Le Royaume-Uni et l'Europe : in and out », Revue internationale et stratégique, vol. 3, no. 91, 2013, p. 151-161.

COURCELLE Thibault, « Les réticences de l'opinion publique britannique face à l'intégration et aux réformes européennes ", Les cahiers de Framespa [En ligne], n 14, 2013, consulté le 15 mars 2018. URL : https://journals.openedition.org/framespa/2556.

CROWLEY John, « Le Royaume-Uni, le Commonwealth et l'Europe », Politique européenne, vol. 2, no. 6, 2002, p. 36-52.

DAVIS Richard, «The Anglo-French Relationship as Seen through British Political Cartoons from the Third to the Fifth Republic ", Revue LISA/LISA e-Journal : Littératures, histoire des idées, images et sociétés du monde anglophone [En ligne], vol. 1, no. 1, 2003, p. 55-74, consulté le 15 mars 2018. URL : http://journals.openedition.org/lisa/3118.

DELPORTE Christian, GERVEREAU Laurent et MARÉCHAL Denis (dir.), Quelle est la place des images en histoire?, Paris, Nouveau monde éditions, 2008.

DELWIT Pascal, «Le parti travailliste face aux communautés européennes (1979-1992) : de l'autarcie à l'ouverture », Politique européenne, vol. 2, no. 6, 2002, p. 74-89.

GERVEREAU Laurent, Voir, comprendre, analyser les images, Paris, Éditions la Découverte, 2004.

HOWORTH Jolyon, «Y a-t-il un dialogue franco-britannique sur l'Europe ? ", Politique étrangère, no. 4 , hiver 2005, p. 823-832.

HOWORTH Jolyon, « La Grande-Bretagne et l'Europe : de la résistance à la rancœur », Politique étrangère, no. 2, été 2010, p. 259-271.

KEMNITZ Thomas Milton, « The Cartoon as a Historical Source », The Journal of Interdisciplinary History, vol. 4, no. 1, 1973, p. 81-93.

LA SERRE (de) Françoise, « De Gaulle et la candidature britannique aux communautés européennes », Histoire, économie et société, vol. 13, no. 1, 1994, p. 131-142. 
LEQUESNE Christian, « La Grande-Bretagne et la construction européenne : anatomie d'une relation conflictuelle », Revue ATALA [En ligne], no. 13, 2010, p. 225-232, consulté le 15 mars 2018. URL : https://www.lycee-chateaubriand.fr/revue-atala/wp-content/uploads/sites/2/2010/10/ Atala13Lequesne.pdf.

LIDDLE Roger, The Europe Dilemma: Britain and the Drama of European Integration, Londres, I.B. Tauris, 2014

MILLAT Gilbert (dir.), Approches iconographiques de la civilisation britannique : images de conflits, conflits d'images, Rennes, Presses Universitaires de Rennes, 2002.

MILLAT Gilbert, « Satire graphique et enracinement national : le dessin de presse britannique », Revue française de civilisation britannique [En ligne], vol. 13, no. 4, 2006, consulté le 4 octobre 2018. URL : https://journals.openedition.org/rfcb/1647.

MILLAT Gilbert, « Le dilemme de l'intégration européenne », in Le déclin de la Grande-Bretagne au XXe siècle dans le dessin de presse, Paris, L'Harmattan, 2008, p. 151-176.

PARR Helen, « Anglo-French Relations, Detente and Britain's Second Application for Membership of the EEC, 1966-1967 », in LUDLOW N. Piers (dir.), European Integration and the Cold War: OstpolitikWestpolitik, 1965-1973, Londres, Routledge, 2007, p. 81-104.

PEYREFITTE Alain, C'était de Gaulle, vol. 1, Paris, Fayard, 1997.

SCHNAPPER Pauline, La Grande-Bretagne et l'Europe : le grand malentendu, Paris, Presses de Science Po, 2000 .

SERGEANT Jean-Claude, Les médias britanniques, Paris, Ophrys-Ploton, 2004.

SHARP Alan et STONE Glyn (dir.), Anglo-French Relations in the Twentieth Century: Rivalry and Cooperation, Londres, Routledge, 2000.

TOULMIN Vanessa et POPPLE Simon (dir.), Visual Delights Two: Exhibition and Reception, Eastleigh, John Libbey Publishing, 2005, p. 145-146.

WALAZEK Helen, The Best of Punch Cartoons, Londres, Prion, 2008, p. 8-13.

YEGANEH CARY-PERNON Niaz, «La politique étrangère britannique au début de la guerre froide : le cas de la crise de Berlin 1948-1949 ", thèse de doctorat en civilisation britannique soutenue sous la direction de Madame la professeure Anne-Marie MOTARD, Université Paul-Valéry Montpellier 3, 2017, p. 253-265.

\section{NOTES}

1. Par exemple, BELL Michael Hett, France and Britain, 1940-1994: The Long Separation, Londres, Routledge, 1997 ou SHARP Alan et STONE Glyn (dir.), Anglo-French Relations in the Twentieth Century : Rivalry and Cooperation, Londres, Routledge, 2000.

2. LA SERRE (de) Françoise, «De Gaulle et la candidature britannique aux communautés européennes ", Histoire, économie et société, vol. 13, n 1, 1994, p. 131-142 ; HOWORTH Jolyon, « Y at-il un dialogue franco-britannique sur l'Europe?", Politique étrangère, no.4, hiver 2005, p. 823-832 ; PARR Helen, «Anglo-French Relations, Detente and Britain's Second Application for Membership of the EEC, 1966-1967 », in LUDLOW N. Piers (dir.), European Integration and the Cold War: Ostpolitik-Westpolitik, 1965-1973, Londres, Routledge, 2007, p. 81-104.

3. DAVIS Richard, «The Anglo-French Relationship as Seen through British Political Cartoons from the Third to the Fifth Republic ", Revue LISA/LISA e-Journal : Littératures, histoire des idées, images et sociétés du monde anglophone [En ligne], vol. 1, no. 1, 2003, p. 55-74, consulté le 15 mars 
2018. URL: http://journals.openedition.org/lisa/3118 ou MILLAT Gilbert, "Le dilemme de l'intégration européenne ", in Le déclin de la Grande-Bretagne au XXe siècle dans le dessin de presse, Paris, L'Harmattan, 2008, p. 151-176.

4. KEMNITZ Thomas Milton, "The Cartoon as a Historical Source », The Journal of Interdisciplinary History, vol. 4, no. 1, 1973, p. 81-82.

5. WALAZEK Helen, The Best of Punch Cartoons, Londres, Prion, 2008, p. 8-13.

6. BBC [En ligne], 24 mars 1992, « On this Day », « Punch ends 150 years of satire », consulté le 4 avril 2018. URL: http://news.bbc.co.uk/onthisday/hi/dates/stories/march/24/ newsid_2531000/2531249.stm.

7. SERGEANT Jean-Claude, Les médias britanniques, Paris, Ophrys-Ploton, 2004, p.114-115 et TOULMIN Vanessa et POPPLE Simon (dir.), Visual Delights Two : Exhibition and Reception, Eastleigh, John Libbey Publishing, 2005, p. 145-146.

8. Les dessins peuvent être consultés sur Punch Cartoon Library, hébergée par TopFoto sur http:// www.topfoto.co.uk. Sur TopFoto, dans "rechercher", il suffit d'écrire le titre des dessins et Punch. Si plusieurs dessins s'affichent, il faut ajouter l'année de la parution et/ou le nom de l'artiste. Nous mentionnerons ci-après seulement le nom de l'artiste, le titre du dessin tel qu'il apparaît sur le site et la date de sa parution originelle dans Punch. Les dessins ont été consultés les 26, 27 et 28 mars 2018. La date de l'accès aux sources ne sera plus indiquée ultérieurement.

9. MILLAT Gilbert (dir.), Approches iconographiques de la civilisation britannique: images de conflits, conflits d'images, Rennes, Presses Universitaires de Rennes, 2002; MILLAT Gilbert, "Satire graphique et enracinement national: le dessin de presse britannique ", Revue française de civilisation britannique [En ligne], vol.13, $\mathrm{n}^{\circ} 4$, 2006, consulté le 4 avril 2018. URL: https:// journals.openedition.org/rfcb/1647. Voir également GERVEREAU Laurent, Voir, comprendre, analyser les images, Paris, Éditions la Découverte, 2004 ou DELPORTE Christian, GERVEREAU Laurent et MARÉCHAL Denis (dir.), Quelle est la place des images en histoire?, Paris, Nouveau monde éditions, 2008.

10. MILLAT, Approches iconographiques, p. 10.

11. ILLINGWORTH Leslie Gilbert, «Pont de la concorde», 17/04/1950. https:// punch.photoshelter.com/image?

\&_bqG=0\&_bqH=eJxtkF1rwyAUhn9Nc5ObZhAYBS.snoazJVr8KLgb6dLSD0LC2sK2fz9PKFvYJnh83vf4Klr08HGA.hTr7vFl9_nWHa8Vf3_QqxA 1Iok2GqiBW5B3uZ5qq41jhqvnbHxq5EqyW2JvwUSUzNM3nJ_Op9K89h0eUmuDxnleR16BEoE2ZVEsI6aDU_SO_hvN6gcbQi4cu.

631_aYbcZ0NVZB9Qv7vnZ4\&GI_ID=

12. Ibid.

13. LIDDLE Roger, The Europe Dilemma: Britain and the Drama of European Integration, Londres, I.B. Tauris, 2014, p. 19-20.

14. YEGANEH CARY-PERNON Niaz, «La politique étrangère britannique au début de la guerre froide: le cas de la crise de Berlin 1948-1949", thèse de doctorat en civilisation britannique soutenue sous la direction de Madame la professeure Anne-Marie MOTARD, Université PaulValéry Montpellier 3, 2017, p. 253-265.

15. ILLINGWORTH, «Go on, it's warmer in than out. », 10/05/1961. https:// punch.photoshelter.com/image?

\&_bqG=0\&_bqH=eJxtUG1LwzAQ_jXpl6BshcI26Ic0ybrTLpUknfRT6HSuG7WFrTrx15srQ4sayN3zck8C19w8X2bNfrldQZS51YZ91PtPV78s3.V 76rTUx1shnQ6VI71C1Xtg8g-\&GI_ID=

16. MANSBRIDGE Norman, "I suspect you of driving under the influence of America.»,
$11 / 10 / 1967$.
https://punch.photoshelter.com/image?

\&_bqG=0\&_bqH=eJxtUFtrwyAY_TXNixS6QhgUfLDqyrc0SVHTkrxImtgmXZeOXDb276ehbGGb4PFcPAofvGQfWf5YDcEyqITcp34xPx7KebYj 2NyNSh1.0.oM_\&GI_ID=

17. PEYREFITTE Alain, C'était de Gaulle, vol. 1, Paris, Fayard, 1997, p. 283.

18. CHAOUAD Robert, "Le Royaume-Uni et l'Europe: in and out", Revue internationale et stratégique, vol. 3, n 91, 2013, p. 152-155. 
19. FAWKES Walter Ernest [TROG], "That's an attractive parcel, sir - now let's examine the contents. ", 30/06/1971. L'artiste signant ses dessins sous le nom de Trog, nous nous y référerons ainsi ci-après.

20. TROG, « The summit », 18/10/1972.

21. Par exemple, ALEXANDRE-COLLIER Agnès, «Le phénomène eurosceptique au sein du parti conservateur britannique », Politique européenne, vol. 2, nº 6, 2002, p. 61.

22. TROG, "How high this time?", 12/05/1971 ou TROG, "Now we can get it finished.", 04/08/1971.

23. MANSBRIDGE, «Leap frog », 23/01/1963 ou MANSBRIDGE, « I suspect you », op. cit.

24. Ibid. et TROG, "If on the other hand, we do have a referendum - and it favours entry..." ", 19/04/1972.

25. DELWIT Pascal, «Le parti travailliste face aux communautés européennes (1979-1992): de l'autarcie à l'ouverture », Politique européenne, vol. 2, n 6, 2002, p. 74-75.

26. LANGDON David, « They're not the terms I'd use myself, but I presume you're referring to our French, German and Italian partners. », 23/05/1979; LANGDON, " Times like this I wish I knew enough French to butt in with "We didn't think about linkage in 39". ", 26/05/1982; PYNE Kenneth, «I tell you ! No one's gonna take away my British identity! », 05/12/1990 ; PYNE, «It's no good - I just can't seem to think of myself as a European yob. », 29/01/1992.

27. LANGDON, "There, in essence, is the story of EEC. », 06/08/1969 et MAHOOD Kenneth, "I'll have a large portion of whatever the EEC has a mountain of. », 09/11/1977.

28. MAHOOD, «I'll have a large portion ", op. cit.

29. HARPUR Merrily, «We get up at six o'clock every morning to milk the EEC agricultural subsidies. », 07/05/1980.

30. BIRKETT Peter, "The European court has quashed your hanging, drawing and quartering sentence. You're to be hung, drawn and decimated. », 02/03/1983 ; LANGDON, «I must admit the pressure is on for the small independent airline. », 12/08/1987 ; LANGDON, « Personally, I'm dead against a single European currency. ", 10/07/1991.

31. LANGDON, «There, in essence ", op. cit. et LANGDON, « Times like this », op. cit.

32. Par exemple, SCHNAPPER Pauline, La Grande-Bretagne et l'Europe: le grand malentendu, Paris, Presses de Science Po, 2000, p. 183.

33. COURCELLE Thibault, « Les réticences de l'opinion publique britannique face à l'intégration et aux réformes européennes », Les cahiers de Framespa [En ligne], n 14, 2013, consulté le 15 mars 2018. URL : https://journals.openedition.org/framespa/2556.

34. LANGDON, «They're not the terms ", op. cit.

35. LANGDON, « Times like this », op. cit.

36. ILLINGWORTH, « Go on », op. cit.; ILLINGWORTH, « Harold Macmillan as Cindrella, sitting by the hearth as the ugly sisters, Charles de Gaulle and Conrad Adenauer, go to the Common Market ball. », 12/09/1962 ; ILLINGWORTH, " "Grandma" de Gaulle : "Come a little nearer, dearie." ", 24/02/1965; MANSBRIDGE, «I suspect you », op. cit.

37. LEQUESNE Christian, «La Grande-Bretagne et la construction européenne : anatomie d'une relation conflictuelle", Revue ATALA [En ligne], no. 13, 2010, p. 228, consulté le 15 mars 2018. URL : https://www.lycee-chateaubriand.fr/revue-atala/wp-content/uploads/sites/2/2010/10/ Atala13Lequesne.pdf.

38. MANSBRIDGE, « Anyone got a plan? », 06/02/1963 ou ILLINGWORTH, « Harold Macmillan as Cindrella », op. cit.

39. ILLINGWORTH, « Harold Macmillan as Cindrella », op. cit.

40. ILLINGWORTH, «"Grandma" de Gaulle », op. cit.

41. MONAR Paul after John TENNIEL, "Would not, could not, would not, could not join the dance. ", 11/05/1990. Il s'agit d'une allusion au dixième chapitre du livre, intitulé « Le quadrille des homards ». Le Griffon et la Tortue y enseignent à Alice à danser le quadrille. 
42. ALEXANDRE-COLLIER, op. cit., p. 64.

43. MANSBRIDGE after LOW 1940, « Very well, alone - if necessary! », 01/02/1967.

44. LOW David, "Very well, alone», The Evening Standard, 18/06/1940, in The British Cartoon Archive de l'Université du Kent à Cantorbéry [En ligne], consulté le 26 mars 2018. URL :

https://archive.cartoons.ac.uk/GetMultimedia.ashx?

$\mathrm{db}=$ Catalog\&type $=$ default\&fname $=$ LSE2791.jpg

45. MANSBRIDGE, "Great Britain is left off the Common Market bus. ", 26/11/1958 ; MANSBRIDGE, «Let's talk this thing over like civilized human beings ", 15/03/1961; MANSBRIDGE, « She'll never make it with all that lot. », 23/11/1966.

46. MANSBRIDGE, « Great Britain is left off », op. cit.

47. Évoquant le projet qui deviendra l'Association européenne de libre-échange en janvier 1960.

48. LOW, «L'Europe - c'est moi!", The Evening Standard, 19/11/1948, in Centre virtuel de la connaissance sur l'Europe de l'Université du Luxembourg [En ligne], consulté le 26 mars 2018. URL : https://www.cvce.eu/obj/

cartoon_by_low_on_general_de_gaulle_s_european_idea_19_november_1948-en-

cdbf03c3-5fc3-4b85-b6d8-40fef7b312b1.html.

49. MANSBRIDGE, «Let's talk», op. cit. et ILLINGWORTH, «Help! Er... I mean au secours!", 19/09/1962.

50. À l'exception de Nessie dans un dessin d'Illingworth en 1961 et outre John Bull, les autres emblèmes nationaux utilisés renvoient à St Georges et au prototype du soldat de la Seconde Guerre mondiale. Par exemple, ILLINGWORTH, «Go on », op. cit.; MANSBRIDGE after LOW 1940, « Very well, alone », op. cit. ; TROG, « Forward ! , 13/10/1971 ; TROG after LOW, « Very well, then - alone ", 10.04/1974.

51. LANGDON, "Oh, do stop it. We've got until 1992 before the EEC abolishes duty-free.", 13/05/1988.

52. LANGDON, «There, in essence ", op. cit. et LANGDON, « Times like this », op. cit.

53. TROG, « Forward! », op. cit.

54. MANSBRIDGE, «Let's talk», op. cit.; MANSBRIDGE, « Export handicap. Between 1953 and 1960 export prices in Britain rose by ten per cent, in Germany by two per cent ; in the Netherlands, they fell by one per cent, and in Switzerland by four per cent. », 28/06/1961; MANSBRIDGE, " Anyone got », op. cit.; TROG, « Nearly there », 07/07/1971 ; MAHOOD, « Change of occupation », 14/07/1971.

55. ILLINGWORTH, « "Grandma" de Gaulle », op. cit. ; MANSBRIDGE, « I suspect you », op. cit. ;

McLACHLAN Edward, "Harold Wilson climbs through a giant "Non" to gain entry to the European Common Market », 26/02/1969.

56. TROG, «Battle for Europe », 20/10/1971; TROG, «If on the other hand », op. cit.; TROG after LOW, «Very well », op. cit. ; TROG, « Punch front cover », 17/11/1076 ; LANGDON, « Ideally, what I'd like if we could have got it, is just simply social membership of EEC. », 13/12/1973.

57. MANSBRIDGE, « Anyone got [...]», op. cit.

58. Par exemple, HOWORTH Jolyon, «La Grande-Bretagne et l'Europe : de la résistance à la rancœur ", Politique étrangère, no. 2, été 2010, p. 260-262.

59. DELWIT, op. cit., p. 74-89.

60. Sur ce point, voir MANSBRIDGE, «Try sideways, missus, try sideways!», 24/08/1960 ; MANSBRIDGE, "Let's talk», op. cit.; MANSBRIDGE, "Now try to read the small type ", 15/08/1962 ; ILLINGWORTH, « Help! », op. cit. ; ILLINGWORTH, « Back to school », 26/09/1962 ; MANSBRIDGE, «She'll never», op. cit.; MANSBRIDGE after LOW 1940, «Very well», op. cit.; MANSBRIDGE, «I suspect you », op. cit.

61. MANSBRIDGE, «She'll never», op. cit.; MANSBRIDGE after LOW 1940, "Very well », op. cit.; MANSBRIDGE, «I suspect you », op. cit. 
62. CROWLEY John, "Le Royaume-Uni, le Commonwealth et l'Europe ", Politique européenne, vol. 2, no. 6, 2002, p. 36-52.

63. MANSBRIDGE, « Let's talk », op. cit. ; ILLINGWORTH, « Help ! », op. cit. ; ILLINGWORTH, « Back to ", op. cit.

64. ILLINGWORTH, « Help ! », op. cit.

65. « Help! Er... I mean au secours ! », ibid.

66. Par exemple, MANSBRIDGE, « Leap frog », op. cit. ; TROG, « How high », op. cit.; TROG, « Now we can ", op. cit.

67. ILLINGWORTH, «Harold Macmillan as Cindrella », op. cit.; ILLINGWORTH, «"Grandma" de Gaulle », op. cit. ; MONAR after John TENNIEL, « Would not », op. cit.

68. MANSBRIDGE, "Great Britain is left off », op. cit.; TROG, « How high », op. cit.; TROG after John TENNIEL, «Picking up another pilot », 03/01/1973 et MONAR after John TENNIEL, « Would not », op. cit.

69. TROG, "Edward Heath and Georges Pompidou at their 1971 summit with the ghosts of Winston Churchill and Charles de Gaulle watching over them », 19/05/1971.

70. ALEXANDRE-COLLIER, op. cit., p. 53-73.

71. MANSBRIDGE, «If any of you know cause, or just impediment, why these seven should not be joined... », 23/05/1962.

72. Ibid.

73. MANSBRIDGE, « Let's talk », op. cit.

74. Ibid.

75. CROWLEY, op. cit., p. 36-52.

76. MCLACHLAN, « Harold Wilson climbs », op. cit. ; MANSBRIDGE, « Export handicap », op. cit. ; ILLINGWORTH, « Go on », op. cit.

77. TROG, « Just married », 19/01/1972 ; TROG, « Now we can », op. cit. ; TROG after John TENNIEL, «Picking up », op. cit.

78. TROG, « The summit », op. cit.

79. HOWORTH, « La Grande-Bretagne », op. cit., p. 259-265.

\section{RÉSUMÉS}

Cet article analyse les relations franco-britanniques dans les dessins politiques de Punch Magazine traitant du thème de la construction européenne de 1950 à 1992. Il s'articule autour de trois axes. Le premier se réfère aux acteurs qui incarnent ces relations. Le deuxième correspond aux références culturelles auxquelles de nombreux dessins font allusion, orientant ainsi la lecture pour y déceler un sens implicite partagé entre les dessinateurs et les observateurs. Enfin, la troisième partie examine la manière dont les rapports franco-britanniques sont esquissés pour étudier le regard que Punch porte sur la politique européenne du Royaume-Uni. Considérant que ces illustrations politiques témoignent d'une représentation médiatique, il s'agit de comprendre comment leur élaboration peut façonner une mémoire visuelle et symbolique.

This article analyses Franco-British relations through Punch Magazine's political cartoons dealing with European integration from 1950 to 1992. It consists of three parts. The first focuses on the personalities embodying the relations; the second investigates the cultural references leading readers to decipher implicit meanings to be shared with the cartoonists; finally, the third part 
examines how the representation of the Franco-British relations in Punch helps convey its perception of the European policy of the United Kingdom. This article argues that the study of the political cartoons in Punch conjures up the production of a visual and symbolic memory of some aspects of British foreign policy.

\section{INDEX}

Keywords : Punch Magazine, Franco-British relations, European integration, political cartoons, visual and symbolic memory

Mots-clés : Punch Magazine, relations franco-britanniques, construction européenne, dessins politiques, mémoire visuelle et symbolique

\section{AUTEUR}

\section{NIAZ CARY-PERNON}

Études Montpelliéraines du Monde Anglophone, EA 741, Université Montpellier 3 - Paul-Valéry, 34199 Montpellier Cedex 5

Professeure d'anglais à l'ENSCM, Niaz Cary-Pernon est docteure en civilisation britannique et rattachée à l'Unité de recherche «Études Montpelliéraines du Monde Anglophone ». Utilisant l'approche interprétative de Mark Bevir, elle a rédigé une thèse sur la politique étrangère britannique au début de la guerre froide. Elle y étudie, plus particulièrement, les relations angloallemandes et la crise de Berlin 1948-1949. 